\title{
Absorption and Scattering of Light by Small Particles
}

\author{
CRAIG F. BOHREN \\ Associate Professor of Meteorology \\ The Pennsylvania State University \\ DONALD R. HUFFMAN \\ Professor of Physics \\ The University of Arizona
}

A Wiley-Interscience Publication

JOHN WILEY \& SONS 


\section{Contents}

\section{PART 1-BASIC THEORY}

\section{Chapter 1. Introduction, 3}

1.1 Physical Basis for Scattering and Absorption 3

1.2 Scattering by Fluctuations and by Particles 4

1.3 Physics of Scattering by a Single Particle 7

1.4 Collections of Particles 9

1.5 The Direct and Inverse Problem 9

Notes and Comments 11

Chapter 2. Electromagnetic Theory, 12

2.1 Field Vectors and the Maxwell Equations 12

2.2 Time-Harmonic Fields 14

2.3 Frequency-Dependent Phenomenological Coefficients 15

2.4 Spatial Dispersion $\quad 22$

2.5 Poynting Vector 23

2.6 Plane-Wave Propagation in Unbounded Media 25

2.7 Reflection and Transmission at a Plane Boundary 30

2.8 Reflection and Transmission by a Slab 36

2.9 Experimental Determination of Optical Constants 41

2.10 The Analogy Between a Slab and a Particle 42

2.11 Polarization 44

Notes and Comments $\quad 56$

Chapter 3. Absorption and Scattering by an Arbitrary Particle, 57

3.1 General Formulation of the Problem 57

3.2 The Amplitude Scattering Matrix 61

3.3 Scattering Matrix 63

3.4 Extinction, Scattering, and Absorption 69

Notes and Comments $\quad 81$ 
Chapter 4. Absorption and Scattering by a Sphere, 82

4.1 Solutions to the Vector Wave Equations 83

4.2 Expansion of a Plane Wave in Vector Spherical Harmonics 89

4.3 The Internal and Scattered Fields 93

4.4 Cross Sections and Matrix Elements 101

4.5 Asymmetry Parameter and Radiation Pressure 119

4.6 Radar Backscattering Cross Section 120

4.7 Thermal Emission 123

4.8 Computation of Scattering Coefficients and Cross Sections 126 Notes and Comments 129

Chapter 5. Particles Small Compared with the Wavelength, 130

5.1 Sphere Small Compared with the Wavelength 130

5.2 The Electrostatics Approximation 136

5.3 Ellipsoid in the Electrostatics Approximation 141

$\begin{array}{ll}5.4 \text { Coated Ellipsoid } & 148\end{array}$

5.5 The Polarizability Tensor 150

5.6 Anisotropic Sphere 152

5.7 Scattering Matrix 154

Chapter 6. Rayleigh-Gans Theory, 158

6.1 Amplitude Scattering Matrix Elements 158

6.2 Homogeneous Sphere 162

6.3 Finite Cylinder 163

Notes and Comments 165

Chapter 7. Geometrical Optics, 166

7.1 Absorption and Scattering Cross Sections 166

7.2 Angular Distribution of the Scattered Light: Rainbow Angles 174

7.3 Scattering by Prisms: Ice Crystal Haloes 178

Notes and Comments

\section{Chapter 8. A Potpourri of Particles, 181}

8.1 Coated Sphere

8.2 Anisotropic Sphere 184

8.3 Optically Active Particles 185

8.4 Infinite Right Circular Cylinder 194

8.5 Inhomogeneous Particles: Average Dielectric Function 213

8.6 A Survey of Nonspherical Particles, Regular and Irregular 219 Notes and Comments 


\section{PART 2-OPTICAL PROPERTIES OF BULK MATTER}

Chapter 9. Classical Theories of Optical Constants, 227

9.1 The Lorentz Model

9.2 The Multiple-Oscillator Model 244

9.3 The Anisotropic Oscillator Model 247

9.4 The Drude Model 251

9.5 The Debye Relaxation Model 259

9.6 General Relationship Between $\epsilon^{\prime}$ and $\epsilon^{\prime \prime} \quad 265$

Notes and Comments 267

Chapter 10. Measured Optical Properties, 268

10.1 Optical Properties of an Insulating Solid: $\mathrm{MgO} \quad 268$

10.2 Optical Properties of a Metal: Aluminum 271

10.3 Optical Properties of a Liquid: Water 273

10.4 A Comment on the Magnitude of $k \quad 279$

10.5 Validity of Bulk Optical Constants in Small-Particle

10.6 Summary of Absorption Mechanism and Temperature Effects 281

Notes and Comments 283

PART 3-OPTICAL PROPERTIES OF PARTICLES

Chapter 11. Extinction, 287

11.1 Extinction $=$ Absorption + Scattering 287

11.2 Extinction Survey 289

11.3 Some Extinction Effects in Insulating Spheres 295

$\begin{array}{ll}11.4 \text { Ripple Structure } & 300\end{array}$

11.5 Absorption Effects in Extinction 305

11.6 Extinction Calculations for Nonspherical Particles 310

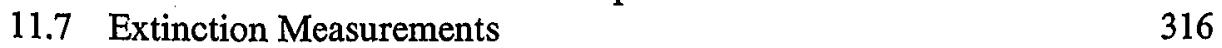

11.8 Extinction: A Synopsis 323

Notes and Comments 324

Chapter 12. Surface Modes in Small Particles, 325

12.1 Surface Modes in Small Spheres 326

12.2 Surface Modes in Nonspherical Particles 342

\begin{tabular}{ll}
12.3 & Vibrational Modes in Insulators \\
\hline
\end{tabular}

12.4 Electronic Modes in Metals 369

Notes and Comments $\quad 380$ 
Chapter 13. Angular Dependence of Scattering, 381

13.1 Scattering of Unpolarized and Linearly Polarized Light 381

13.2 Techniques of Measurement and Particle Production 389

13.3 Measurements on Single Particles 394

13.4 Some Theoretical and Experimental Results 397

13.5 Particle Sizing 403

13.6 Scattering Matrix Symmetry 406

13.7 Measurement Techniques for the Scattering Matrix 414

13.8 Some Results for the Scattering Matrix 419

13.9 Summary: Applicability of Mie Theory 427

Notes and Comments $\quad 428$

Chapter 14. A Miscellany of Applications, 429

14.1 The Problem of Optical Constants 430

14.2 Atmospheric Aerosols 434

14.3 Noctilucent Clouds 448

14.4 Rainfall Measurements with Radar 454

14.5 Interstellar Dust 457

14.6 Pressure Dependence of Intrinsic Optical Spectra

14.7 Giaever Immunological Slide 469

14.8 Microwave Absorption by Macromolecules 472

\section{APPENDIXES COMPUTER PROGRAMS}

Appendix A. Homogeneous Sphere, 477

Appendix B. Coated Sphere, 483

Appendix C. Normally Illuminated Infinite Cylinder, 491

References, 499

Index, 521 